\title{
Modelo físico y simulación computacional de un colector fotovoltaico-térmico pv/t con circulación de agua
}

Gonzalo José Durán ${ }^{1}$, Miguel Angel Condorí2 ${ }^{(*)}$

\section{RESUMEN:}

En este trabajo se desarrolla un modelo téorico para analizar un colector fotovoltaico - térmico (FV/T). El modelo integra los aspectos térmicos y eléctricos, analizando la conversión fotoeléctrica de radiación solar y la cogeneración de calor a baja temperatura mediante la refrigeración con agua en la parte posterior del colector. Se realizó para un día tipo la simulación numérica bajo Simusol de un colector de 40 celdas y $80 \mathrm{Wp}$, planteando dos escenarios. En el primero se estudió el desempeño del módulo sin circulación de agua, y en el segundo, con circulación de flujo. En la simulación del colector con circulación de agua se encontró que la potencia eléctrica se incrementa en hasta un $15 \%$ respecto al caso de estudio sin refrigeración.
Palabras clave: Simulación, cogeneración, PVT, calentador solar de agua, panel fotovoltaico

ABSTRACT: In this work a theoretical model is developed to analyze a photovoltaic - thermal collector $(F V / T)$. The model integrates the thermal and electrical aspects, analyzing the photoelectric conversion of solar radiation and the cogeneration of heat at low temperature by cooling with water at the rear of the collector. The numerical Simusol simulation of a collector of 40 cells and $80 \mathrm{WP}$ was carried out for a day, proposing two scenarios. In the first, the performance of the module without water circulation was studied, and in the second, with flow circulation. In the simulation of the collector

(*) Instituto de Investigaciones en Energía no Convencional (INENCO, UNSa - CONICET) Universidad Nac. de Salta- Av. Bolivia 5150 - A4408FVY Salta, Argentina - FAX 543874255489 - e-mail: gonzalo.jose.duran@gmail.com ${ }^{1}$ Investigador Asistente CONICET.

${ }^{2}$ Investigador Independiente CONICET - Director INENCO 
with water circulation it was found that the electric power increases by up to 15\% with respect to the case of study without refrigeration.

Keywords: Simulation, cogeneration, $P V T$, solar water heater, photovoltaic panel

\section{INTRODUCCION:}

La potencia entregada por los paneles fotovoltaicos depende de la radiación solar incidente, de la temperatura de la celda y de la resistencia de carga. En general, el fabricante provee datos de la tensión en circuito abierto Voc, la corriente de corto circuito Isc, los valores de tensión y corriente para máxima potencia $V m p$ e Imp, y los coeficientes de variación con la temperatura de los valores anteriores, referidos a condiciones estándar de radiación $(1000 \mathrm{~W} / \mathrm{m} 2)$ y temperatura de celda $\left(25^{\circ} \mathrm{C}\right)$.

En un panel, una fracción de la radiación solar incidente es convertida en electricidad por efecto fotoelectrico, y el resto de la energía es disipada como calor, contribuyendo al incremento en temperatura de la celda. Como consecuencia, la eficiencia eléctrica cae (Platz et. al., 1997 Kalogirou 2006; Chow, 2010), pues la eficiencia de las celdas depende de las condiciones ambientales, y la temperatura de operación juega un papel fundamental.

Desde hace tiempo que se viene estudiando diseños teóricos y experimentales para el enfriamiento de los paneles FV. Una de las soluciones más eficientes se basa en combinar un panel FV estándar con un un intercambiador de calor con circulación de líquidos en un sistema híbrido fotovoltaico - térmico $(\mathrm{PV} / \mathrm{T})$. Con ello, es posible producir en simultáneo agua caliente de baja temperatura y electricidad, con una mejor eficiencia de conversión electrica. Un trabajo de Zondag et. al. (2003) reporta que, comparando resultados de un módulo convencional con un panel $\mathrm{PV} / \mathrm{T}$ sin cubierta, y con un panel PV/T con cubierta, se obtienen eficiencias anuales medias de $6.6 \%, 7.2 \%$ y $7.7 \%$.

En este trabajo se analiza un modelo teórico de colector $\mathrm{PV} / \mathrm{T}$, basado en la resolución simultánea de un circuito térmico y un circuito de cinco parámetros característicos que representa la conversión fotoeléctrica. Se aplica el modelo a un colector de dimensiones y materiales simulares al estudiado en Spertino et. al. 2016, resolviendo la simulación numérica en base horaria mediante Simusol, y se discuten los resultados obtenidos al estudiar el panel FV sin circulación de agua, con circulación de agua, y en el caso de operación al caudal óptimo de agua que maximice la eficiencia de conversión eléctrica. 


\section{Modelo Eléctrico:}

El modelo eléctrico utilizado se basa en el estudio del conjunto de celdas mediante el

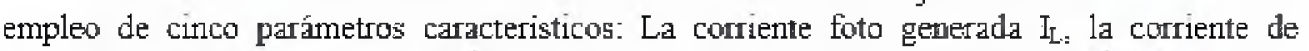

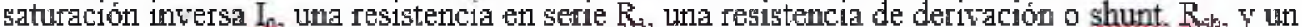
parámetro $a_{2}$ que representa el factor de idealidad modificado del diodo, que depende de la temperatura de la celda. La figura representa un esquema del circuito equivalente de una celda fotoroltaica en base al modelo de los cinco parametros.

La principal rentaja de este modelo, es que permite estimar ell comportamiento del panel a partir de los dator que brinda el fabricante en la hoja de especificaciones. Utiliza la radiación solar absorbida y la temperatura de celda combinadas en ecuaciones semi empiricas para predecir la curva I-V de la celda (y la potencia). Fue talidado rigurosamente contra datos experimentrales encontrando coincidencias muy buenas (De Soto et al, 2006).

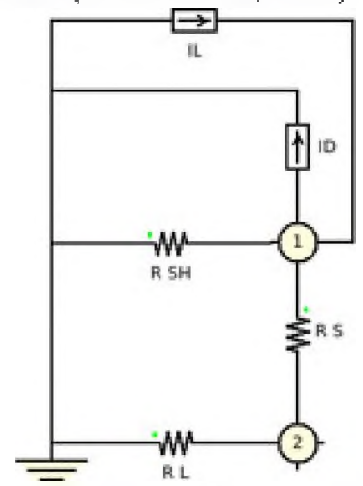

Figura 1: Circuito equibalente del modelo de los cinco parámetros para un panel fotovoltaico

Puesto que se necesita conocer cinco parámetros, se tiene que utilizar cinco condiciones distintas. Usualmente se encuentran en base a los tres puntos de la curra I-T del panel protistos por el fabricante (los que representan las condiciones de circuito abierto, corto circuito y máxima potencia), la condición de que la derivada de la potencia respecto al voltaje es cero en el punto de maxima potencia, y el coeficiente de variación del voltaje de circuito abierto con la temperatura (uin) en condiciones de corto circuito, circuito abierto y máxima potencia.

Las siquientes son las ecuaciones que representan con buena aproximación este comportamiento, halladas por Townsend y presentadas en Duffie y Beckman, 2013.

$$
\begin{aligned}
& \frac{a}{a_{r+f j}}=\frac{T_{c}}{T_{r+\rho^{\prime}}}(1) \\
& I_{D}=I_{0}\left[e^{\frac{V+I R_{S}}{a}}-1\right] \\
& R_{s}=\frac{a \cdot m\left(1-\frac{I_{m p p}}{I_{L}}\right)=v_{m p}+v_{O C}}{I_{m p p^{*}}}
\end{aligned}
$$

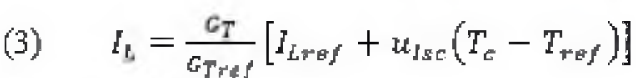

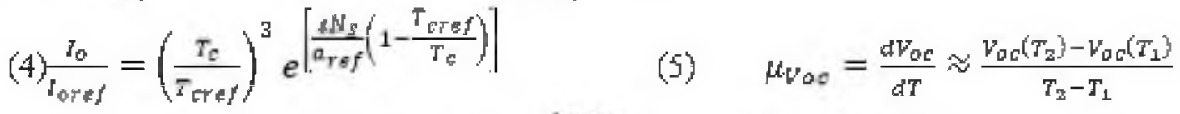

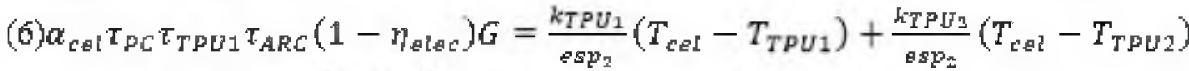

$$
\begin{aligned}
& \text { (7) } \quad \eta_{e l}=\frac{P_{\max S C}}{G_{S C} A_{e f}}\left(1-\gamma_{m p}\left(T_{c e l}-T_{S C}\right)\right)(8)
\end{aligned}
$$


$\varepsilon=$ encrgia de banda prohibida ( $\mathrm{eT}_{\mathrm{r}}^{\mathrm{T}}$ )

$\mathrm{T} 1, \mathrm{~T} 2=$ temperaturas cercanas a $\mathrm{Tc}(\mathrm{K})$

$\mathrm{N}_{\mathrm{s}}=$ nümero de celdas en serie,

Vlel $=$ eficiencia de conversion elléctrica
$\mathrm{T}_{\mathrm{c}}=$ temperatura de la celda $(\mathrm{K})$

$\mathrm{G}=$ imadiancia (Wim 2)

Subindice ref $=$ de referencia

Subindice $\mathrm{SC}=$ de referencia

\section{Modelo Térmico:}

El modelo termico se basa en calcular, mediante los balances de calor correspondientes, las temperaturas de las interfaces entre las distintas capas del colector. Se analiza el modelo térmico para dos casos: modulo $F V$ sin circulacion de agua, y FV con circulación de agua.

La figura 2 es un esquema de sección transersal del modulo FT, donde se muestran los flujos de calor de los que intercienent en los balances (azul). Ein rojo, los nodos de temperaturas de cada interfaz, y en negro, los nombres y coeficientes representativos en cada sección.

Panel FV sin circulación de agua: Para el analisis se realizarán las siguientes hipótesis

1. - Flujo de calor a través del modulo PV estacionario.

2.- Se considerara unidimensional al flujo de calor, pa que cualqujer gradiente significatito de temperatura existirá predominantemente en la dirección normal a las celdas $F$.

3.- Las conductividades térmicas de las capas del módulo se consideraran constantes.

4.- Se supondràn despreciables las resistencias de contacto en las interfaces.

5. - En la celda FW el espesor de la celda es despreciable, y de temperatura uniforme.

6.- Se consideraran despreciables las absortancias solares de la capa de poliuretano termoplástico (TPU) o o y y de las superficies de policarbonato atro

\section{Balance de calor en cubierta:}

Interviene la perdida de calor al ambiente por confeccion por viento, la perdida de calor por radiacion a cielo abierto, y la conducción en el policarbonato de la cubienta.

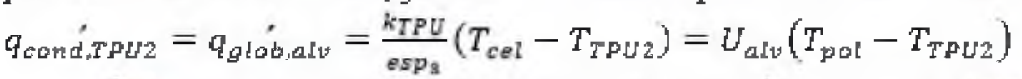

$k_{R C}$ representia el coeficiente conductivo del policarbonato. La convescion por viento se ha calculado por Watmuff et. al 1977 , donde $v$ es la relocidad del riento:

$$
h_{\text {iquan }}=2 . s+3 v
$$

La femperatura de cielo se ha estimado mediante Notton et al.,2005:

Balance de calor en la interfaz policarbonato - TPUI

En la interfaz cubierta-TPU, el flujo de calor es netamente conductiro

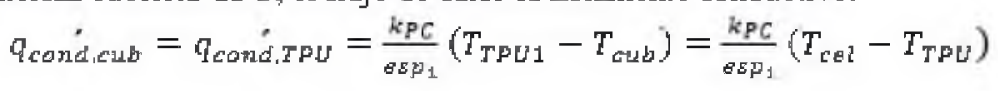

Balance de calor en la celda:

En la celda, el recubrimiento anti reflectivo permite considerar a la celda como una superficie opaca de tal manera que $a_{k e}=1$. La radiación absorbida en la celda está determinada por la porción de la radiación incidente sobre la cubierta, quitando las pérddas ópticas.

$$
\alpha_{c e 1} \tau_{P C} \tau_{T P U 1} T_{A R C}\left(1-n_{e l e c}\right) G=\frac{k T P U_{1}}{s s p_{2}}\left(T_{c e I}-T_{T P U_{1}}\right)+\frac{k_{T P U_{2}}}{B s p_{3}}\left(T_{c e l}-T_{T P U 2}\right)
$$

(13)

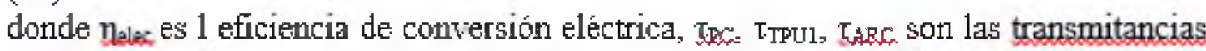
de la cubierta de policarbonato, placa de TPU, y del tratamiento antireflective, $a_{\text {cel }}$ es la absortancia de la celda, $y$ iston representa la conductioidad de la placa de TPU.

Balance de calor en la interfac TPU2-policarbonato alveolar: 


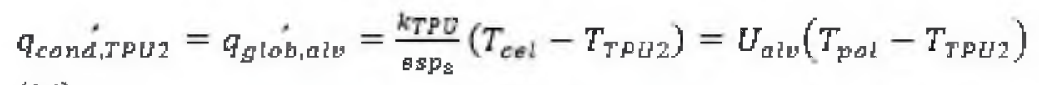

(14)

En el ducto de policarbonato alvelar no esta circulando agua, solo se tiene aire. La resistencia térmica en el ducto es çonvectiva y siguiendo a Spertino et al (2016), se puede calcular como un coeficiente global, de valor aproximado $\mathrm{U}_{\text {als }}=6 \mathrm{~W} / \mathrm{m} \mathrm{m}^{2} \mathrm{~K}$.

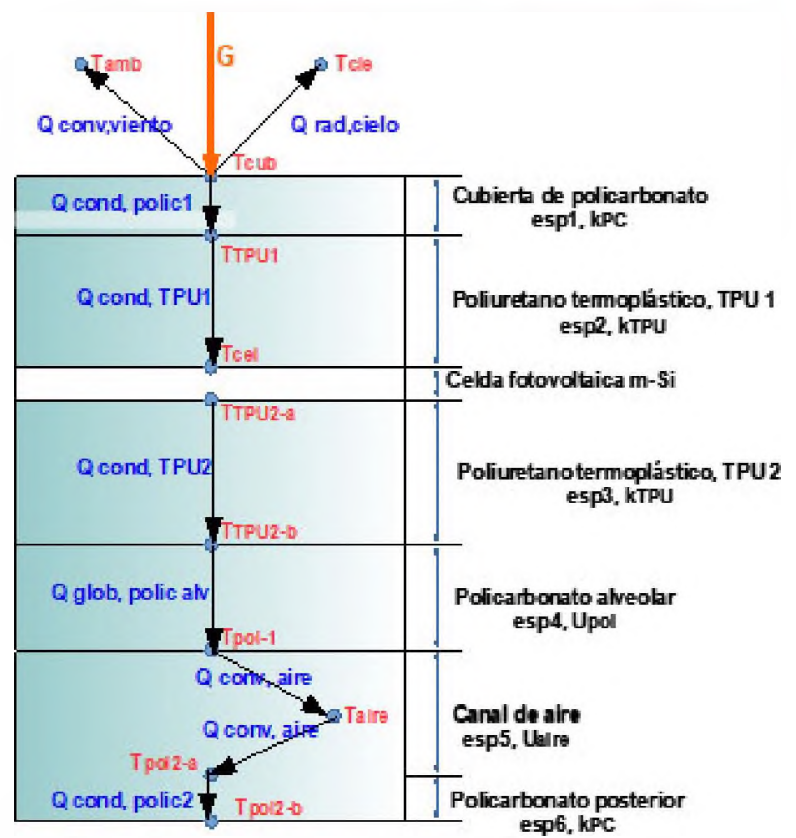

Figura 2: Sección transversal del modulo Fvi sin circulación de agua.

Balance de calor entre la cara inferior del policarbonato aiveolar y el ducto de arre:

El balance de calor entre la capa de policarbonato alreolar y el ducto de aire inferior es:

$\left.T_{\text {air }}\right)(16)$

$$
q_{g l o b, a l t}=q_{g l o b_{1} a t r e}=U_{a l v}\left(T_{p a l}-T_{T P U 2}\right)=U_{a i r}\left(T_{c u b i a 1 f}-\right.
$$

Luego, en la interfaz policarbonato alveolar - camara de aire, puede utilizarse la misma expresion que en el balance anterior donde toma unt valor equiparable $\mathrm{U}_{\text {sine }}=12 \mathrm{~W}^{2} \mathrm{~m}^{2} \mathrm{~K}$

Balance de calor en la interfor entre el aire del ducto y la cara intema de la cubierta posterior:

$\left.T_{p 012-b}\right)$

$$
q_{g l a b, a 1 r^{\prime}}=q_{\text {condid,pol2 }}=U_{\text {air }}\left(T_{\text {air }}-T_{\text {pol2-a }}\right)=\frac{k_{P C}}{e s p_{6}}\left(T_{p o l 2-a}-\right.
$$

\section{Panel FV con circulación de agua}

Se wa a suponer que el incremento de temperatura de agua se produce en la dirección del flujo (a lo largo del panel), por intercanbio de calor con las secciones superiores del colector. Las hipótesis a considerar son similares a las tomadas para el módulo sin refrigerar.

Se van a despreciar los efectos de conducción en las laminas de policarbonato y la sección de TPU2, y entre las láminas de policarbonato y la cara adyacente al ducto de aire.

\section{Balance de calor en el agua:}

higu representa el coeficiente convectivo que se calcula en base al numero de Nusselt, Nus, el cual se considero fijo para un flujo lasmitiar. 
$\left.T_{p o[1}\right)(18)$

$$
q_{\text {canviggua1 }}=q_{\text {cattudagua }}=h_{\text {agua }}\left(T_{\text {TPU2 } 2}-T_{\text {agua }}\right)=h_{\text {agta }}\left(T_{\text {agua }}-\right.
$$

Incropera 2006, indica que en estas condiciones Nu toma el talor 3,608 para recintos cuadrados. Como Reynolds se calcula en base al diametro hidráulico de los alceolos, se tiene que $\mathrm{h}_{\text {ngua }}$ toma talores entre $240 \mathrm{y} 260 \mathrm{~W} / \mathrm{mK}$, para temperaturas de flujo entre $20 \mathrm{y} 70^{\circ} \mathrm{C}$.

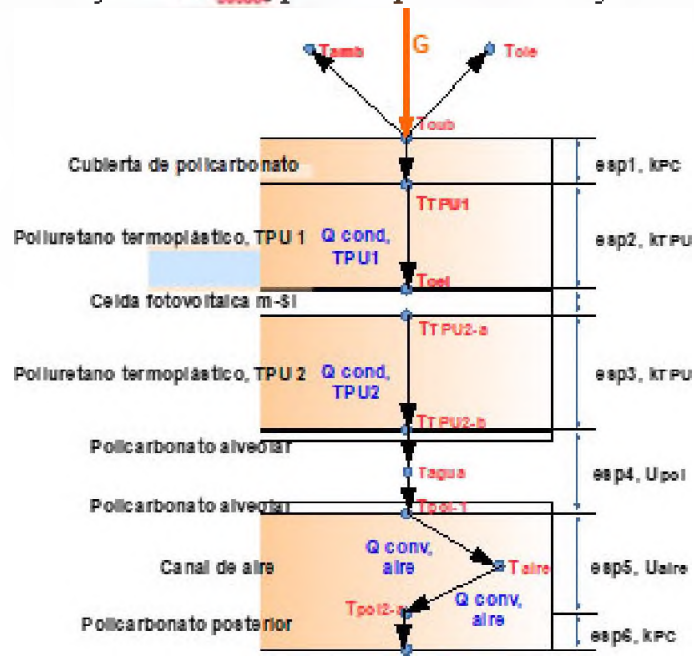

Figura 2: Esquema de secciones para el colector con circulacion de agua en su parte posterior.

\section{SITLLACIÓN DEL COLECTOR PVTT BAJO SIRUSOL}

Lisualmente los fabricantes de paneles no proveen información sobre las propiedades físicas de las capas que componen sus módulos Fv, ya que se trata muchas rieces de datos confidenciales. Debido a ello, se decidio seguir a Sperting et. al., 2016 , en donde se describen las propiedades terrmicas de las diversas capas de un módulo comercial, de marca no detallada para preservar la confidencialidad de la información descrita. Se decidio simular un módulo $F$ V de 40 celdas de silicio mono cristalino: $80 \mathrm{Wpg}$ de potencia, y de $1,47 \mathrm{~m} \mathrm{x} 0,6 \mathrm{~m}$. Se supuso inclinacion de $30^{\circ}$ sobre la horizontal, y como localidad, Salta, Argentina.

Se realizó la simulación suponiendo un tiempo de 12 horas, suponiendo condiciones climáticas de un dia claro de inrierno en la localidad de Salta. Argentina (Lat: $24^{\circ} 47^{\prime} \mathrm{L} 8^{\prime \prime S}$, Long. $\left.65^{\circ} 24^{\prime} 38^{\prime \prime} \mathrm{O}, 1180 \mathrm{~ms} . \mathrm{nm}\right)$. La temperatura antiente se varió entre una minima de $17^{\circ} \mathrm{C}$. mäxima de $23^{\circ} \mathrm{C}$ : con pico a hs 13.00 . Los valores de radiación directa: difusa de cielo y difusa de suelo se generaron mediante el software GEOSOL, para el dia 21 de junio, en Salta, con $30^{\circ}$ de pendiente y albedo de 0,3, mediante el metodo de estimación de dia claro de Liu - Jordan. En base a ello, la radiacion global sobre plano de colector presenta un pico de $950 \mathrm{~W}^{2} \mathrm{~m}^{2}$, a horas 12:00. For último, se consideró un valor de velocidad de viento promedio de 2 mis.

\begin{tabular}{|c|c|c|c|}
\hline Propientad & Talor & & \\
\hline Coud de la cubierta de policarbonato ( $\{\mathrm{C}\}, \mathrm{kPC}$; & $0,2 \mathrm{BDE}$ & Eqponor de la placa tasera de PC, epp 6 & $4 \mathrm{~s} 10-5 \mathrm{~m}$ \\
\hline Couductiridad de plaea de TPU lardu & $0,2 \mathrm{~W} / \mathrm{mK}$ & Firesiternia de shomst Roh $(\Omega)$ & 200 \\
\hline Egpesor de la cubiett fromtal de PC, exp] & $0,375 \times 10$-35 & Resistencia de cortociruito, $R=(\Omega)$ & $7,64 \times 10-3$ \\
\hline Eqpesar de la placa fiontal de TPUl ,esp2 & $10-3 m$ & Factor de idesindad del duado, $\operatorname{man}(\mathrm{V})$ & 1,249 \\
\hline Eopear de la placa posterior de TPU2, enp? & $1,5 \times 10-3 \mathrm{~m}$ & 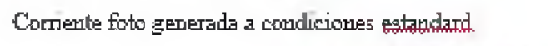 & 4,73 \\
\hline Enp de la placa de poliesubonato alyedolar, esp4t & $0,6 \times 10-3 m$ & Corrente de diodo a condicioner estanciard. Iph SC(A) & $9,2 \times 10-8$ \\
\hline
\end{tabular}




\begin{tabular}{|c|c|c|c|}
\hline Slltura del ducto de agur & $8.8 \times 10-5 \mathrm{~m}$ & 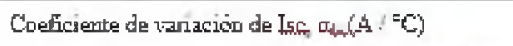 & 0,038 \\
\hline Althra del camal de alre, agps & $10-2 m$ & Corrente misama, a condiciones estandard $\operatorname{Imn}(\mathrm{A})$ & 4,48 \\
\hline Eat mixima, a condiciones estandard Prux(W) & 87,61 & Tención misima, a sondiciones estandard Vome(V) & 19,07 \\
\hline
\end{tabular}

Tabla 1: Parámetros fisicos y eléctricos del colector analizado.

Simulacion de colector sin refrigeracion:

Se realizo una primer simulación del colector, sin circulación de agua, como referencia. Simulacion del colector con cjpulación de agua:

Se discretizó el rolumen del colector para hallar los gradientes térmicos en sus distintas superficies. La discretización se realizo tomando cinco trolimenes de control, cada uno de dimensiones $0,6 \mathrm{~m}$ de ancho, 0,284 $\mathrm{m}$ de largo, $y$ alto equivalente a la suma de los espesores $y$ alturas de canales. Se supuso conectado al colector a un tanque de agua de 20 litros. convenientetrente aislado.

Los ensayos se centraron en hallar el caudal óptimo que maximice la potencia eléctrica entregada por el colector. Una vez hallado el caudal, se realizo una sequnda corrida a caudal constante; cotn objeto de conocer los gradientes de temperatura en las superficies del colector.

\section{RESULTADOS:}

Simulacion simpio de agua:

En funcionamiento sin refrigeracion la máxima potencia entregada por el panel es de 74 W, al mediodia solar. En ese mismo instante, el roltaje y la coniente entregados son $19,28 \mathrm{~W}$ y 3.86 A. Se puede obserar, comparando con la tabla de propiedades del panel que estos raleres son menores que los de condición de máxina potencia. En el mismo instante la temperatura de celda es máxima, con un salto de $30^{\circ} \mathrm{C}$ respecto a la temperatura ambiente

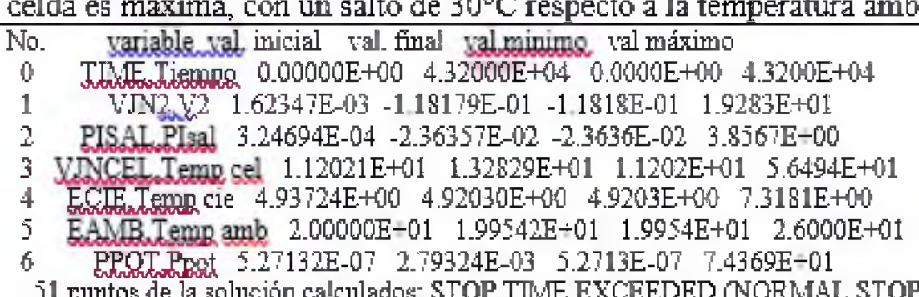

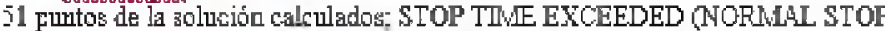

Tabla 2ui Resumen de resultados de simulación sin refrigeración.
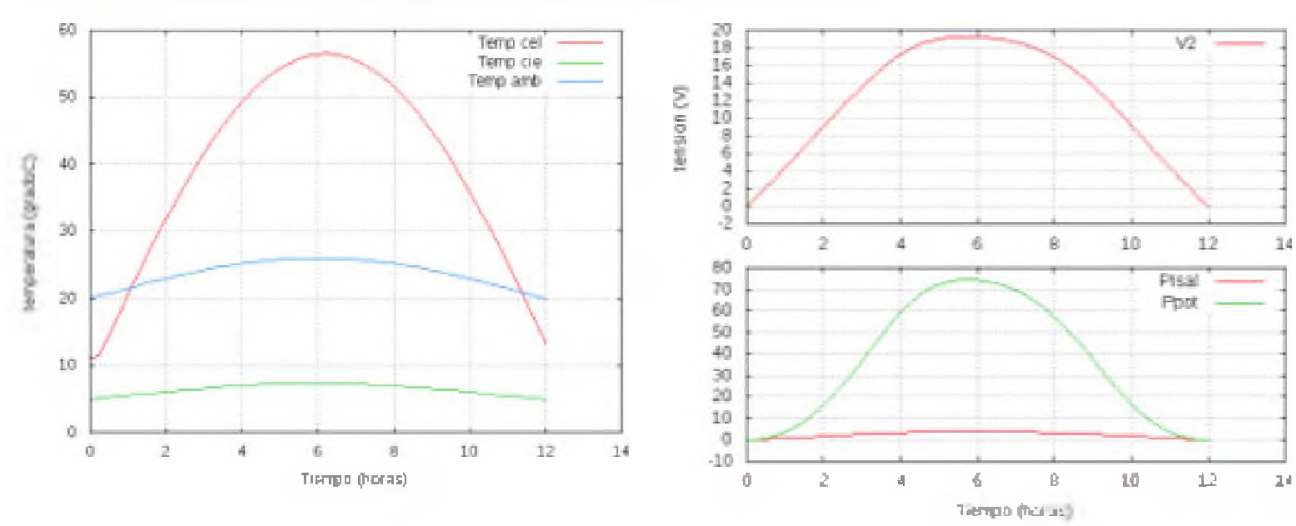

Figura 3: Temperaturas de celda, ambiente y cielo. Voltaje de salida y potencia entregada por el panel. Simulacion sin flujo de agua.

Simulacion del colector PVT con agua. Caudal optimo.: 
A continuación se reseñarán los resultados obtenidos en las simulaciones con caudal de flujo forzado, con valores de 1 y 15 litroshora. En esta corrida se evalúan los resultados de

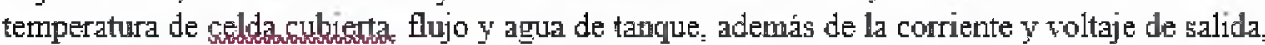
Y la potencia entregada por el colector, donde:

1. temp 5t3: nodo 5 (temp celda) del tercer wolumen de control (volumen central)

2. teme 3t3: nodo 3 (teme de cubietta) del tercer volumen de control (central)

3. temp 9t3: nodo 9 (tenog de flujo de agra) del tercer tolumen de control (central)

4. temp tan: nodo de temperatura de agua en tanque de resersa de 20 litros.

5. temp 14t5: fondo del colector, del tercer volumen de control (extremo de salida)

6. ธ2: roltaje de salida, en roltios. Ppot: potencia de salida del panel, en ratios

7. Ppisal: Representa la coniente de salida del panel, en amperes.

Los resultados indican que el caudal para los mejores resultados eléctricos es 1 lih Aunque no se muesran eft este trabajo, los resultados a caudales nuas pequeños no representat mejora sustancial. Por otro lado, el imcremento en caudales lleva a una disminución máxima en la potencia entregada a mediodia solar de $92.9 \mathrm{~W}$ a $82.3 \mathrm{~W}$ aprox $10 \mathrm{~W}$

\begin{tabular}{|c|}
\hline 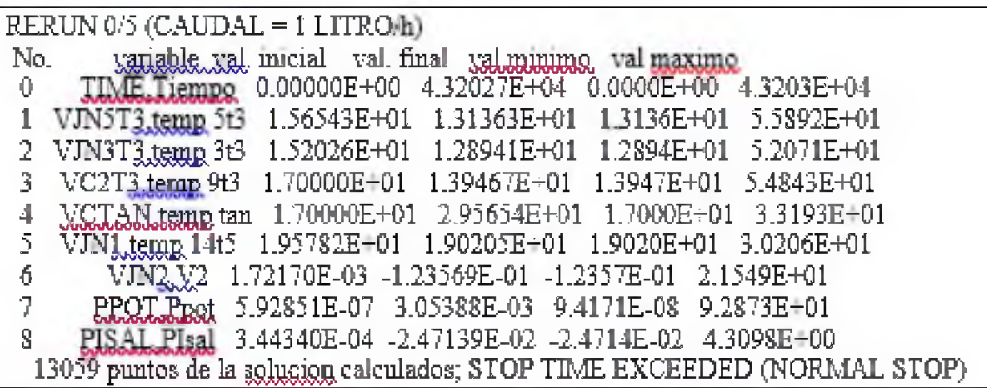 \\
\hline Tabla 3: Resunthan de resultados con caudal de agua de 1 litro. \\
\hline 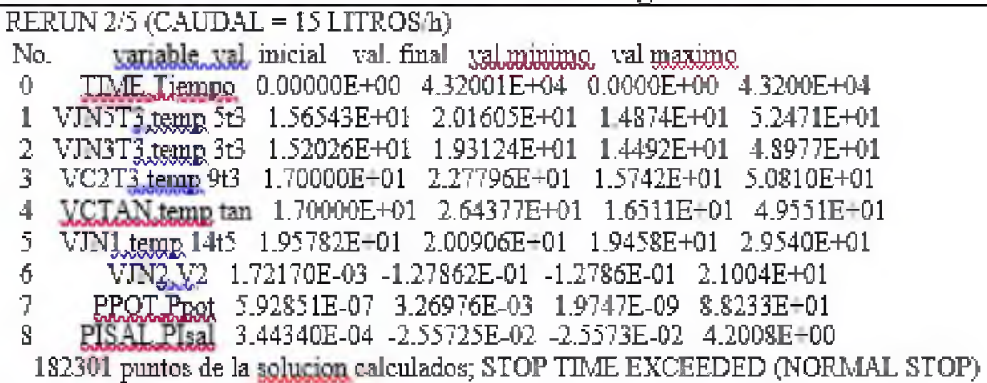 \\
\hline
\end{tabular}

Tabla 4: Resumen de resultados con flujo de agua de 15 litros.
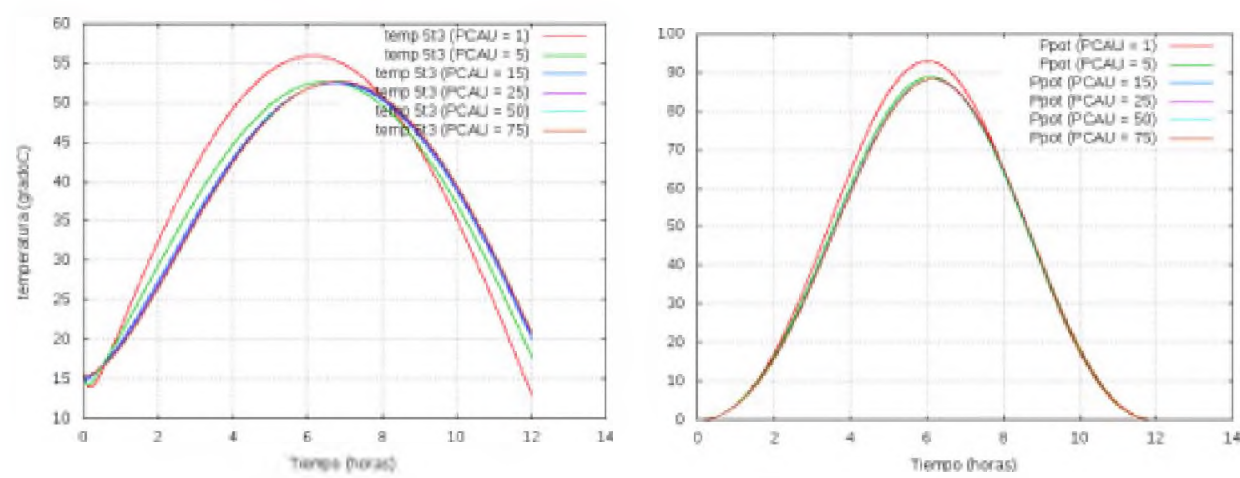

Figura 5: Tecperatura de celda a distintong caudales. Figura 6: Foteacia entrengada a distintos caudales. 


\section{CONCLUSIONES:}

Se ha estudiado un modelo termo eléctrico de colector FViT basado en la resolución zimultánea de un circuito de nodos de temperatura, $y$ otro circuito que representa el comportamiento eléctrico. Se resuelve el modelo mediante una simulación numérica bajo Simusol, considerando un dia de sol de invierno en la ciudad de Salta, Argentina. Se plantean dos escenarios: el primero simulando el colector en modo $\mathrm{FW}$, sin la circulación de agua. En esa simulación se halla que la máxima potencia entregada por e. panel es de $74 \mathrm{~W}$ al mediodía solar. En ese mismo instante, el roltaje y la corriente entregados son 19,28 V y $3,86 \mathrm{~A}$. Comparando con la tabla de propiedades del panel, estos valores son menores que los de máxima potencia. En el mismo instante la temperatura de celda es máxima, con un salto de $30^{\circ} \mathrm{C}$ respecto a la temperatura ambiente. Como siguiente paso, se evalúa el colector en funcionamiento como colector FVT: con el objeto de hallar el caudal de agua que maximice la conversion eléctrica. Para ello se corren simulaciones parametrizadas por el caudal de agua circulante, con valores de $1,5,15,25,50$ y 75 th. Los resultados simulados indican que el caudal para el cual se obtienen los mejores resultados eléctricos es $1 \mathrm{lih}$, valor para el cual se pasa de una potencia máxima de $74.4 \mathrm{~W}$ a $92.9 \mathrm{~W}$, 10 que representa un incremento de hasta $25 \%$ en potencia máxima. A mismos valores, la temperatura de celda disminuye de $56.5^{\circ} \mathrm{C}$ a $55.9^{\circ} \mathrm{C}$ a con lo cual se puede considerar que no se registra un incremento sustancial de temperatura a pesar del incremento en potencia entregada. Para finalizar, se describe el comportamiento del colector al caudal mas favorable, $1 \mathrm{lh}$, observando que los gradientes de temperatura en el panel son pequerios fuera de la zona del panel en contacto con la entrada de agua fría. Se preteent realizar ensayos prácticos en un prototipo experimental, con el objetivo de ralidar el modelo descrito.

\section{REFERENCLAS}

Chow, T.T. 2010. A review on photovoltaict thermal hybrid solar technology. Appl. Energy 87, 365-379.

Sperting et. al. Thermal-electrical model for energy estimation of a water cooled photovoltaic module Solar Energy 133 (2016) 119-140.

Platz et al. 1997. Hybrid collectors using thin-filn technology. Proceeding of the Photoroltaic Specialists Conference, Conference Record of the Twenty-Sixth IEEE. Anaheim, California pp. 1293-1296.

Kalogirou, S.A.. Tripanagnostopoulos, Y., 2006. Hybrid PV/T solar systems for domestic hot water and electricity production. Energy Convers. Manag. 47, 3368-3382.

De Soto W., Klein S. And Beckman W. (2006). Improvement and ralidation of a model for photoroltaic array performance. Solar Energy 80. pp. 78-88.

Duffie I. A. y Beckman W. A. (2013). Solar Engineering of Thermal Processes, 3 a edición: pp. 768-780. Wiley Interscience, New York 